\title{
miR-24-3p and miR-27a-3p promote cell proliferation in glioma cells via cooperative regulation of MXI1
}

\author{
WEIYI XU ${ }^{1 *}$, MINGFA LIU $^{2 *}$, XUELING PENG ${ }^{1 *}$, PENG ZHOU $^{1}$, JIANWEN ZHOU $^{1}$, \\ $\mathrm{KE} \mathrm{XU}^{2}$, HAIXIONG XU ${ }^{2}$ and SONGSHAN JIANG ${ }^{1}$ \\ ${ }^{1}$ Key Laboratory of Gene Engineering of the Ministry of Education, State Key Laboratory of Biocontrol, \\ School of Life Sciences, Sun Yat-sen University, Guangzhou; ${ }^{2}$ Department of Neurosurgery, \\ Affiliated Shantou Hospital of Sun Yat-sen University, Shantou, P.R. China
}

Received October 9, 2012; Accepted November 29, 2012

DOI: 10.3892/ijo.2012.1742

\begin{abstract}
MicroRNAs (miRNAs) are small, non-coding RNAs which regulate gene expression at the post-transcriptional level. Abnormal expression of miRNAs occurs frequently in tumors. Although the two miRNAs miR-24-3p and miR-27a-3p come from two duplicated gene clusters of miR-23a 27a 24-2 and miR-23b 27b 24-1 which are found to be deregulated in a variety of cancers, the role of cooperation of the two clusters and the function of the two miRNAs in tumors have not been completely characterized. Here, we show that overexpression of miR-24-3p and miR-27a-3p could promote cell proliferation using the MTT assay. By integrated bioinformatic analysis and experimental confirmation, we identified MXI1, which has been found to act as a tumor suppressor gene by affecting c-Myc, as a direct target of miR-24-3p and miR-27a-3p. While targeting the MXI1 3' untranslated region by miR-24-3p or miR-27a-3p, luciferase activity was attenuated. The two miRNAs promote glioma cell proliferation via targeting MXI1 and the experiment was confirmed by the rescue experiments. Furthermore, our results show that two clusters of miR-23a 27a 24-2 and miR-23b 27b 24-1 regulate MXI1 synergistically. These findings reveal, for the first time, the novel functions of cooperation of miR-24-3p and miR-27a-3p from two clusters in promoting cell proliferation through MXI1. Additionally, we observed that miR-27a-3p is upregulated in glioma tissues.
\end{abstract}

Correspondence to: Professor Songshan Jiang, State Key Laboratory of Biocontrol, School of Life Sciences, Sun Yat-sen University, 132 East Waihuan Rd., Rm 312, Higher Education Mega, Guangzhou, Guangdong 510006, P.R. China

E-mail: jiangssh@mail.sysu.edu.cn

Dr Haixiong Xu, Department of Neurosurgery, Affiliated Shantou Hospital of Sun Yat-sen University, 114 Wai Ma Road, Shantou, Guangdong 515041, P.R. China

E-mail: xuhaixiong@yahoo.com

*Contributed equally

Key words: miR-24-3p, miR-27a-3p, MXI1, cell proliferation

\section{Introduction}

Gliomas are the most lethal brain tumors, they account for $\sim 50 \%$ of all central nervous system tumors (1). According to the histopathological and clinical criteria established by the World Health Organization (WHO), tumors are graded on a scale of I to IV, in which glioblastoma multiforme (GBM) is the most common and aggressive form of brain tumor in adult (2), its median survival ranges from 9 to 12 months. The etiology of glioma is largely unknown, despite the advanced surgical, radiation and medical therapies (3). To clarify the genetic mechanisms in glioma pathogenesis has become an important challenge for development of new therapies.

Increasing evidence show that post-transcriptional regulation of gene expression is mediated by microRNAs (miRNAs) playing an important role in a variety of cancers including gliomas (4-7). miRNAs are a class of regulatory small RNAs with 19-24 nucleotides (nt) that enable to downregulate expression of their target genes by partially complementing with targeting mRNAs. The miRNA target site has been considered to be the $3^{\prime}$ untranslated region (3' UTR) of mRNA, however, recent studies have shown that mRNAs may also bind the coding regions or the 5 ' untranslated regions $\left(5^{\prime}\right.$ UTRs) $(8,9)$. Due to the miRNAs being implicated in the pathogenesis of various cancers (6), they are considered to be novel therapeutic targets. Many independent studies provide evidence that miRNAs can act as oncogenes to reduce expression of their target tumor suppressor genes in tumors, or tumor suppressors to produce higher levels of target oncogene expression, leading to neoplasia $(6,10)$.

Two miRNA clusters, miR-23a 27a 24-2 and miR$23 \mathrm{~b} \sim 27 \mathrm{~b} \sim 24-1$ are found to be deregulated in a variety of tumors $(11,12)$. The former mainly produces mature miR-23a-3p, miR-27a-3p and miR-24-3p; the latter mainly produces miR$23 b-3 p, m i R-27 b-3 p$ and $\operatorname{miR}-24-3 p$. miR-24-3p is a master regulator (11). It is reported that miR-24-3p was upregulated consistently during terminal differentiation of hematopoietic cell line into a variety of lineage (13), also during muscle and neuronal cell differentiation $(14,15)$. Several studies have reported that miR-24-3p might function differently in cell proliferation in different cells (12). For example, miR-24-3p was able to inhibit cell proliferation in HeLa cells (16), in contrast, 
it promotes cell proliferation of transforming growth factor $\beta$-treated hepatocellular carcinoma cells ( $\mathrm{HuH7})$, as well as A549 lung carcinoma cells (17). miR-27-3p has been reported to regulate adipogenesis, myeloblasts differentiation (18), skeletal muscle development (19) and osteoblast differentiation (20). In breast cancer cells, miR-27a-3p could enhance the expression of specificity protein $(\mathrm{Sp})$ transcription factors which is overexpressed in tumours where they contribute to the proliferative and angiogenic phenotype by regulating number of angiogenic proteins (21). miR-27a-3p along with miR-27b-3p were upregulated in activated hepatic stellate cells and promoted cell proliferation (22). The two clusters contain three families of miRNAs, although each family of the miRNAs is paralogous, they have complex expression patterns. The miRNAs of miR-23a 27a 24-2 cluster are derived from one primary transcript (23), in contrast, the miRNAs derived from miR-23b 27b 24-1 cluster have different primary transcripts for each pre-miRNA (24). Therefore the miRNA expression patterns vary from different biological conditions (11). miR24-3p was discovered to be upregulated in the glioblastoma cell lines and miR-27a-3p was often upregulated in glioblastoma tissue $(25,26)$. However, the function of these miRNAs in gliomas is still unclear.

MXI1, a member of Mad (Mxi1) family, encodes a protein with 228 amino acids. It can form a heterodimer with the highly stably expressed protein Max to function as the antagonist of c-Myc, a transcription factor belonging to the basic helix-loop-helix-ZIP family, which is essential to cellular growth and development and it is involved in the processes including control of cell proliferation and induction of apoptosis $(27,28)$. The MXI1 gene localizes at chromosome 10q24-q25, deletion of this sequence has been demonstrated in $60-80 \%$ of human glioblastoma tumors (29-31) and in $20-30 \%$ of human prostate tumors $(32,33)$. Numerous studies have shown both MXI1 and MAD can inhibit the function of c-Myc, so that they have been considered to be tumor suppressor genes (28). The loss of MXI1 function might lead to tumor progression $(34,35)$.

Although miR-24-3p and miR-27a-3p derive from two duplicated gene clusters of miR-23a 27a 24-2 and miR-23b 27b 24-1, the roles of the two clusters and the two miRNAs in tumors have not been completely characterized. In this study, both miR-24-3p and miR-27a-3p have been shown to promote glioma cell proliferation. We have identified three miRNAs from the two miRNA clusters which can synergistically regulate MXI1. Furthermore, we have demonstrated that miR-24-3p and miR-27a-3p promoted cell proliferation by directly regulating MXI1, and miR-27a-3p was found significantly upregulate in glioma tissues.

\section{Materials and methods}

Cell culture. Human glioma cell lines (U87 and U251) were purchased from American Type Culture Collection (Manassas, VA, USA). U87 and U251 cells were cultured in DMEM (Invitrogen Life Technologies, USA) containing $10 \%$ fetal bovine serum (FBS, Invitrogen Life Technologies), $100 \mathrm{U} / \mathrm{ml}$ penicillin and $100 \mu \mathrm{g} / \mathrm{ml}$ streptomycin (Invitrogen Life Technologies) at $37^{\circ} \mathrm{C}$ with $5 \% \mathrm{CO}_{2}$.
Human tissue samples. Archival frozen human glioma tissue samples (including a total of 17 tumors and 17 peritumoral tissues) were obtained from Shantou Hospital of Sun Yat-sen University in accordance with the Committee on Human Research approved procedures. All of the samples were obtained with informed consent of the patients and were histologically confirmed. Total-RNA from tissues was isolated by TRIzol (Invitrogen Life Technologies) according to the manufacturer's instructions.

Vector construction and transfection. To express miRNA, genomic fragments containing human miRNA precursors with about 80 bp of flanking sequences in both sides were amplified using the primers listed in Table I and cloned into the modified pLL3.7 vector under the control of the human U6 promoter.

To construct luciferase reporter vectors, full length 3' UTR sequence of MXI1, first half and second half of MXI1 3' UTR or a mutated sequence of the 3' UTR sequence were amplified using the primers listed in Table I and cloned downstream of Renilla luciferase in the psiCHECK-2 vector (Promega, USA). miRNA sensors were constructed according to the reported method (36) by inserting tandem four copies of the complementary sequences of mature miRNAs at the 3' UTR region of the Renilla luciferase in psiCHECK-2 vector.

MXI1-cDNA was obtained from NCBI CDS database (NM_005962.4) and constructed into XhoI and BamHI sites after the CMV promoter of the cDNA expression vector pcDNA-neo, which is reconstructed from pcDNA3.1+. The primers are represented in Table I. miR-24-3p and miR-27a-3p mimics, and the negative control mimics were synthesized and purified by Gene Science \& Health, China.

RNA extraction and real-time quantitative $R T-P C R$. Total-RNA was extracted from frozen brain specimens using TRIzol reagent (Invitrogen Life Technologies) and were reverse-transcribed using ReverTraAce- $\alpha$ transcriptase (Toyobo, Japan). The sequences of the forward and reverse primers for MXI1/c-MYC listed in Table I. The RNA was quantified and checked for purity by spectrophotometry at 260 and $280 \mathrm{~nm}$ and subsequently amplified by PCR using the Taq DNA polymerase (Takara, Japan). The expression of human miR-27a-3p and miR-24-3p was quantitated in human tissues using SYBR ${ }^{\circledR}$ Premix Ex Taq ${ }^{\mathrm{TM}}$ (Tli RNaseH Plus) (Takara). Using U6 RNA as an internal standard and sets of primers were purchased from Gene Science \& Health, China. The comparative $\mathrm{Ct}(\Delta \Delta \mathrm{Ct})$ method was used to determine the expression fold change.

Western blotting. U87 or $293 \mathrm{~T}$ cells were lysed in RAPI lysis buffer (Bioteke, China) and concentration was determined by bicinchoninic acid protein assay kit (Beyotime, China). Heat-denatured protein samples ( $20 \mu \mathrm{g}$ per lane) were loaded onto a $10 \%$ SDS-polyacrylamide gel electrophoresis (PAGE) and transferred to an Immobilon-P membrane (Millipore, USA). The membrane was incubated with a primary goat polyclonal antibody against human Mxil (1:500 dilution) or mouse monoclonal antibody against human $\beta$-actin $(1: 2000$ dilution) and then incubated for $1 \mathrm{~h}$ with a rabbit anti-goat (1:10,000 dilution) or goat anti-mouse (1:10,000 dilution) 
Table I. The primers used in our study.

\begin{tabular}{|c|c|}
\hline Gene name & Primer sequence ( $5^{\prime}$ to $\left.3^{\prime}\right)$ \\
\hline Sensor24 & $\begin{array}{l}\text { S1: TCGAATAACTGTTCCTGCTCCCTGAGCCACGATCTGTTCCTGCTCCCTGAGCCA } \\
\text { AS1: ACGCGTTGGCTCAGGGAGCAGGAACAGATCGTGGCTCAGGGAGCAGGAACAGTTAT } \\
\text { S2: ACGCGTCTGTTCCTGCTCCCTGAGCCATCACCTGTTCCTGCTCCCTGAGCCAC } \\
\text { AS2: GATCGTGGCTCAGGGAGCAGGAACAGGTGATGGCTCAGGGAGCAGGAACAG }\end{array}$ \\
\hline Sensor $23 a$ & $\begin{array}{l}\text { S1: TCGAATAAGGAAATCCCTCTAATGTGATCGATGGAAATCCCTCTAATGTGAT } \\
\text { AS1: ACGCGTATCACATTAGAGGGATTTCCATCGATCACATTAGAGGGATTTCCTTAT } \\
\text { S2: ACGCGTGGAAATCCCTCTAATGTGATTCACGGAAATCCCTCTAATGTGATC } \\
\text { AS2: GATCGTGGCTCAGGGAGCAGGAACAGGTGATGGCTCAGGGAGCAGGAACAG }\end{array}$ \\
\hline Sensor $27 \mathrm{a}$ & $\begin{array}{l}\text { S1: TCGAATAAGCGGAACTTACGACTGTGAACGATGCGGAACTTACGACTGTGAA } \\
\text { AS1: ACGCGTTTCACAGTCGTAAGTTCCGCATCGTTCACAGTCGTAAGTTCCGCTTAT } \\
\text { S2: ACGCGTGCGGAACTTACGACTGTGAATCACGCGGAACTTACGACTGTGAAC } \\
\text { AS2: GATCGTTCACAGTCGTAAGTTCCGCGTGATTCACAGTCGTAAGTTCCGC }\end{array}$ \\
\hline GAPDH & $\begin{array}{l}\text { S: CCCATGTTCGTCATGGGTGT } \\
\text { AS: TGGTCATGAGTCCTTCCACGATA }\end{array}$ \\
\hline MXI1 & $\begin{array}{l}\text { S: ATTCCACTAGGACCAGACTGCACC } \\
\text { AS: GCTGGTGGTACTTATATTGTCCAC }\end{array}$ \\
\hline MYC & $\begin{array}{l}\text { S: TCAAGAGGCGAACACACAAC } \\
\text { AS: GGCCTTTTCATTGTTTTCCA }\end{array}$ \\
\hline MXI1-cDNA & $\begin{array}{l}\text { S: ATGGAGCGGGTGAAGATGATCAAC } \\
\text { AS: TGCACTGTTATGTCATGCTGGGT }\end{array}$ \\
\hline MXI1-cDNA & $\begin{array}{l}\text { S: GACGCTGGATTTTTTTCGGGTAGTGG } \\
\text { AS: CTTACGCACAAGAGTTCCGTAGCTG }\end{array}$ \\
\hline MXI1-3'UTR Full & $\begin{array}{l}\text { S: CTCGAGTAGAACCCAGCATGACATAACAGTG } \\
\text { AS: GGATCCTTCTTCGTTCACAGTTTTTATTTCTTC }\end{array}$ \\
\hline MXI1-UTR1 & $\begin{array}{l}\text { S: CCGCTCGAGGACATAACAGTGCAGGGCAAAATA } \\
\text { AS: CGGGATCCAAACAGCCAGGGGTAAGGTCTC }\end{array}$ \\
\hline MXI1-UTR2 & $\begin{array}{l}\text { S: CCGCTCGAGATTGATAGATCTTTATGTTTAGATAGGGCTGGGCAAG } \\
\text { AS: CGGGATCCTCTTCGTTCACAGTTTTTATTTCTTC }\end{array}$ \\
\hline MXI1-UTR1-MUT & $\begin{array}{l}\text { S: AGGCCAAGGTGACTCGGACAGCAGCATTTTTATTTC } \\
\text { AS: AATGCTGCTGTCCGAGTCACCTTGGCCTGCTAATCT }\end{array}$ \\
\hline MXI1-UTR2-MUT & $\begin{array}{l}\text { S: CCGCTCGAGATTGATAGATCTTTATGTTTAGATAGGGCTGGGCAAG } \\
\text { AS: CGGGATCCTCTTCGTAGTGTCATTTTATTTCTTCCAATTAACTT }\end{array}$ \\
\hline
\end{tabular}

$\mathrm{S}$, sense primer; AS, anti-sense primer.

secondary antibody. The bound antibody was detected with the use of enhanced chemiluminescence detection reagents (Pierce, USA) according to the manufacturer's instructions.

The primary antibody and second antibody of Mxil were bought from Santa Cruz Biotechnology, Inc. (Santa Cruz, CA, USA) and Abcam Inc. While the primary antibody of $\beta$-actin and second antibodies were purchased from Sigma-Aldrich, Inc. and Jackson ImmunoResearch Inc.

Dual luciferase reporter assays. 293T cells $\left(2.5 \times 10^{4}\right)$ in $100 \mu 1$ growth medium were plated in 96-well plates. The next day, the cells were transfected with $100 \mathrm{ng}$ psiCHECK2-MXI1-3' UTR or psiHECK2-MXI1-3' UTR-MUT and 300 ng pre-miRNAs or 40 nM miRNA mimics using Lipofectamine 2000 (Invitrogen Life Technologies) or FuGene (Roche, Switzerland). The cells were harvested $48 \mathrm{~h}$ after transfection and assayed using the
Dual-Luciferase Reporter Assay kit (Promega) according to the manufacturer's instructions. Transfection was repeated in triplicate.

MTT (dimethyl thiazolyl diphenyl tetrazolium) assay. U87 cells were seeded at $3 \times 10^{3}$ cells per well in 96 -well plates. Negative control or miR-24-3p mimics were transfected with Lipofectamine 2000 (Invitrogen Life Technologies) or FuGene HD (Roche) according to the manufacturer's instructions. At different time points $(24,48,72$ and $98 \mathrm{~h})$ post-transfection, MTT reagent $(5 \mathrm{mg} / \mathrm{ml})$ was added directly to the medium and incubated at $37^{\circ} \mathrm{C}, 5 \% \mathrm{CO}_{2}$ incubator for $4 \mathrm{~h}$. Then supernatants were removed and $150 \mu \mathrm{l}$ DMSO was added to dissolve the formazan crystals, and thoroughly mixed for $10 \mathrm{~min}$. Optical densities at $490 \mathrm{~nm}$ were measured using culture medium as a blank. 
Lentivirus packaging. VSV-G pseudotyped lentiviruses were produced by co-transfection of $293 \mathrm{~T}$ cells. One day before transfection, 293T cells were seeded in $100-\mathrm{mm}$ dishes. $2.3 \mu \mathrm{g}$ pMK-VSVG, $5 \mu \mathrm{g}$ pMDL-G/P-RRE, $3.8 \mu \mathrm{g}$ pRSV-REV and $7.6 \mu \mathrm{g}$ miRNA expression vector (pre-miR-24-2 or pre-miR-27a) were mixed and transfected by Lipofectamine 2000 according to the manufacturer's instructions. The cells grew to about $80 \%$ after $72 \mathrm{~h}$ post-transfection, the production medium containing lentivirus was harvested, centrifuged to remove cell debris and viral supernatant was used for infection.

Cell growth curve. U87 stable cell lines were established which expressed miR-24-3p and miR-27a-3p, with empty vector as a control. In day 0,500 cells were seeded in 96-well plates. MTT assay were performed on day 1,3,5 and 7. Absorbance was normalized by day 1 and cell growth curve of U87 stable cells were measured in 7 days.

Statistical analysis. Data are presented as the mean \pm standard deviation (SD) of at least three separate experiments. The data were analyzed using the SPSS 12.0 Windows version software. Statistical analyses were done by analysis of variance or Student's t-test. p-value $<0.05$ was considered statistically significant $\left({ }^{*} \mathrm{p}<0.05 ;{ }^{* *} \mathrm{p}<0.01 ;{ }^{* * *} \mathrm{p}<0.001\right)$.

\section{Results}

miR-24-3p and miR-27a-3p promote cell proliferation in glioma cells. The miR-24-3p and miR-27a-3p have been found to be abundant in glioma cells $(11,26)$. In order to evaluate the role of these two miRNAs in cell proliferation, we first constructed miR-24-3p or miR-27a-3p expression vectors by inserting miRNA precursor containing some flanking sequences at both sides into the pLL3.7 vector. To measure whether our miRNA constructs could efficiently produce mature miRNAs, miRNA sensors were constructed according to the reported method (36) by inserting tandem four copies of the complementary sequences of mature miRNAs at the 3' UTR region of the Renilla luciferase (Rluc) in psiCHECK-2 vector. Co-transfection of each miRNA expression vector (empty vector as control) and cognate sensor followed with dual luciferase assay showed that the relative luciferase ratio declines significantly while comparing with that of the control (Fig. 1A), indicating that our constructs produced mature miRNAs efficiently. Then we performed transient transfection of these vectors containing pre-miR-24-2 and pre-miR-27a into U87 glioma cells, respectively. MTT assay at $72 \mathrm{~h}$ posttransfection showed that overexpression of both pre-miR-24-2 and pre-miR-27a in U87 glioma cells were able to promote the cell proliferation, respectively (Fig. 1B).

To confirm these results, the mimics of the miR-24-3p and miR-27a-3p were performed for the same MTT experiment using the validated constructs we have measured before. It was shown that the mimics have similar effects to the overexpression of the pre-miRNAs (Fig. 1C). Then, we established stable U87 glioma cell lines that overexpress pre-miR-24-2 (U87-pre-miR-24-2) or pre-miR-27a (U87-pre-miR-27a). MTT assay revealed that overexpression of pre-miR-24-2 or premiR-27a significantly increased cell proliferation in seven days (Fig. 1D and E).
Similarly, we performed the experiments in U251 cells. When the two miRNAs were transfected in U251 cells, MTT assay showed that the cell proliferation increased (Fig. 2A). The result indicated that miR-24-3p and miR-27a-3p induced cell proliferation in diverse glioma cell types. To substantiate the role of miR-24-3p and miR-27a-3p in cell proliferation of glioma cells, we inhibited the maturation of miR-24-3p and miR-27a-3p by shRNAs complementary against miR-24-3p and miR-27a-3p (marked as anti-miR-24-3p and anti-miR-27a-3p). We used these shRNAs to test cell proliferation in U251 cells. At $72 \mathrm{~h}$ post-transfection, the level of miR-24-3p and miR-27a-3p decreased significantly when measured by quantitative RT-PCR (qPCR, data not shown) and MTT assay revealed that inhibition of miR-24-3p by anti-miR-24-3p and miR-27a-3p by anti-miR-27a-3p in U251 cells decreased the cell proliferation, respectively (Fig. 2B). Taken together, these results indicated that miR-24-3p and miR-27a-3p promoted cell proliferation in glioma cells.

Three miRNAs from two duplicated gene clusters all target the MXII gene. The two miRNAs, miR-24-3p and miR-27a-3p are produced from two miRNA clusters, miR-23a miR27a miR-24-2 at chromosome 19p13 which is intronic and miR-23b miR-27b miR-24-1 at chromosome 9q22 which is intergenic (Fig. 3A). miR-27a and miR-27b are two paralogous sequences of miR-27 that differ at only one position (22). To investigate how miR-24-3p and miR-27a-3p promote cell proliferation of glioma cells, we predicted miR-24-3p and miR-27a-3p target genes using prediction software including TargetScan, miRanda, PicTar and EIMMo. Among the predicted target genes, miR-24-3p and miR-27a-3p, as well as miR-27b-3p, had been shown to interact with MXI1 gene (Fig. 3B), which is a tumor suppressor gene deleted in $60-80 \%$ of human glioblastoma tumors (29-31). To test the predicted interactions, we first constructed Renilla luciferase reporter vector containing the full 3' UTR of MXI1 gene. Co-transfections with the miRNA expression vector plus the 3' UTR report vector into 293T cells were preformed to detect the interactions. Each miRNA precursor from the clusters miR-23a miR-27a miR-24-2 and miR-23b miR-27b miR-24-1 were tested and we found that pre-miR-24-1, pre-miR-24-2, pre-miR-27a and pre-miR-27b significantly suppressed the luciferase activity (Fig. 3C). Since the miRNA precursors pre-miR-24-2 and pre-miR27a from the same cluster suppressed the luciferase activity more strongly than pre-miR-24-1 and pre-miR-27b which located in the other cluster, and the predicted target sites of miRNA from pre-miR-24-1 and pre-miR-24-2, or miRNAs from pre-miR-27a and pre-miR-27b are identical, we chose the pre-miR-24-2 and pre-miR-27a from the same cluster for further investigation.

To determine which mature miRNA from the two arms of each pre-miRNA might regulate the MXI1 3' UTR, we co-transfected synthetic mimics of each mature miRNA plus reporter vector containing full length of MXI1 3' UTR into 293T cells and measure the luciferase ratio. We found that miR-24-3p and miR-27a-3p mimics were able to reduce significantly the luciferase activity compared with that of the control, however, miR-24-1-5p, miR-24-2-5p and miR27a-5p mimics could not (Fig. 4A), indicating that miR-24-3p and miR-27a-3p can regulate MXI1 via its 3 ' UTR. While 
A

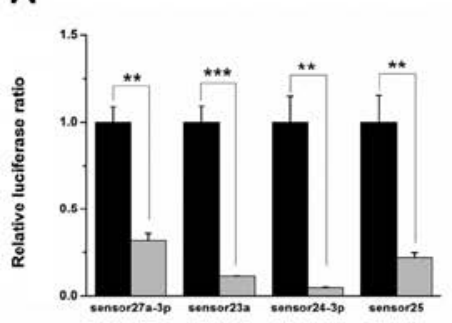

B

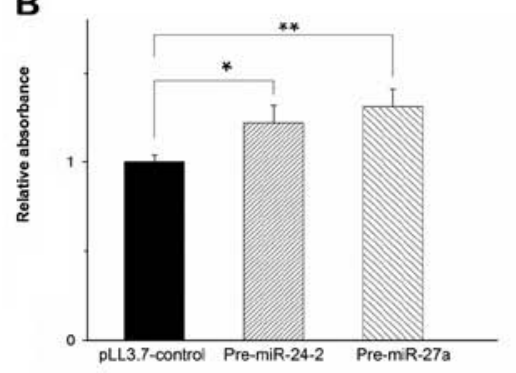

C

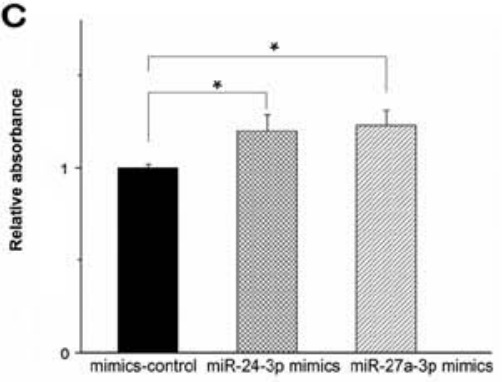

D

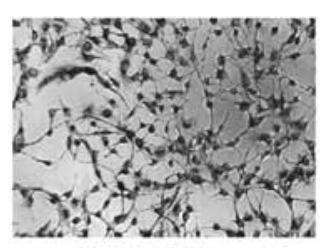

U87-pLL3.7-control

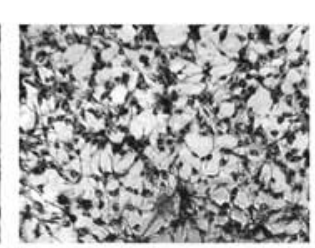

U87-pre-miR-24-2

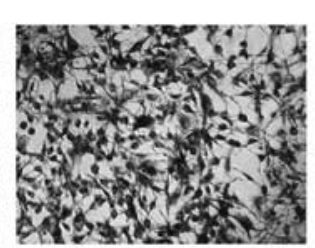

U87-pre-miR-27a

\section{$\mathbf{E}$}

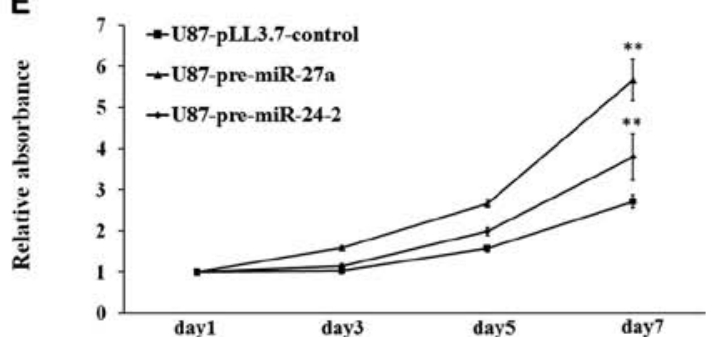

Figure 1. miR-24-3p and miR-27a-3p promote cell proliferation in U87 glioma cells. (A) The pre-miRNA expression vectors (pre-miR-27a, pre-miR-23a, pre-miR-24-2 and pre-miR-25) and cognate miRNA sensors (miR-27a-3p, -23a, -24-3p and -25) were co-transfeted in 293T cells to test whether the construct could express the miRNA. Luciferase assay was measured at $48 \mathrm{~h}$. Values represent the means \pm SD from three independent experiments. Significant differences from the control value are indicated by ${ }^{* *} \mathrm{p}<0.01$ and ${ }^{* * * *} \mathrm{p}<0.001$. (B) U87 cells were transiently transfected with empty vector or pre-miR-24-2 or pre-miR-27a expression vector. After $72 \mathrm{~h}$ of incubation, an MTT assay was performed to determine cell viability. Values represent the means \pm SD. Significant differences from the control value are indicated by ${ }^{*} \mathrm{p}<0.05$ and ${ }^{* *} \mathrm{p}<0.01$. (C) U87 cells were transfected with miRNA (miR-24-3p or miR-27a-3p) or negative control (NC) mimics and the cell growth was investigated by MTT assay at $72 \mathrm{~h}$. Values represent the means \pm SD. Significant differences from the control value are indicated by ${ }^{*}$ p $<0.05$. (D) Image of control (empty vector), pre-miR-24-2 and pre-miR-27a stable cell lines after 7 days cultivation. (E) Pre-miR-24-2 and pre-miR-27a significantly increased cell growth in U87 stable cell lines compared to control group. The maximum rate is at 7 days $\left({ }^{* *} \mathrm{p}<0.01\right)$.
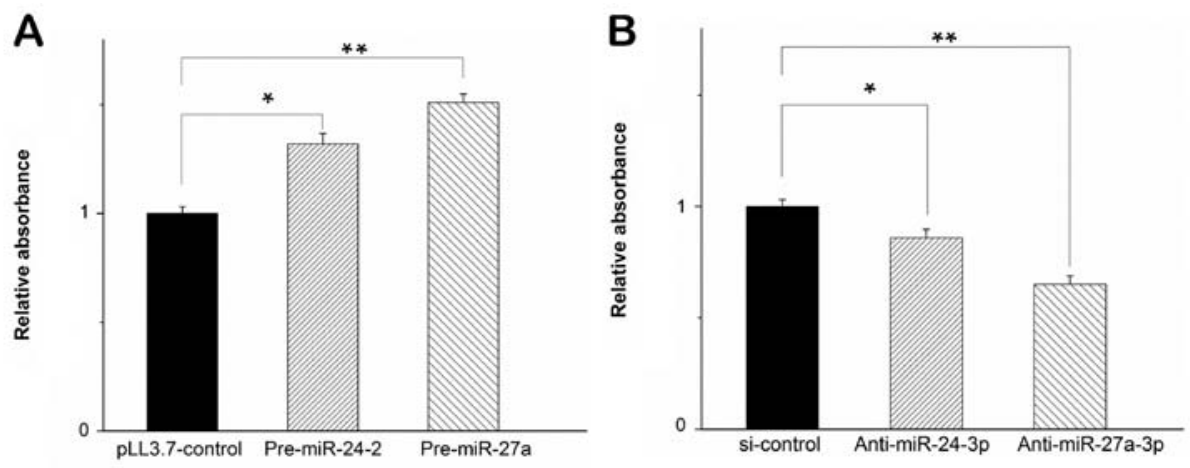

Figure 2. miR-24-3p and miR-27a-3p promote cell proliferation in U251 glioma cells. (A) U251 cells were transiently transfected with empty vector or premiR-24-2 or pre-miR-27a expression vector. An MTT assay was performed after $72 \mathrm{~h}$ of incubation. Absorbance at $570 \mathrm{~nm}$ was normalized to the control to determine cell viability. Values represent the means \pm SD. Significant differences from the control value are indicated by ${ }^{*} \mathrm{p}<0.05$ and ${ }^{* *} \mathrm{p}<0.01$. (B) The growth of U251 cells were inhibited by anti-miR-24-3p and anti-miR-27a-3p and followed the MTT assay after $72 \mathrm{~h}$ of incubation. Values represent the means \pm SD. Significant differences from the control value are indicated by ${ }^{*} \mathrm{p}<0.05$ and ${ }^{* *} \mathrm{p}<0.01$.

measuring the level of endogenous Mxi1 proteins, western blot assay showed that both pre-miR 24-2 and pre-miR-27a consistently and substantially downregulated the expression of MXI1 in 293T cells (Fig. 4B) and U87 cells (Fig. 4D), the mimics of the miR-24-3p and miR-27a-3p showed the same effect in 293T cells (Fig. 4E). However, qPCR analyses showed pre-miR-24-2 and pre-miR-27a did not change the mRNA level of MXI1 gene (Fig. 4C). These results suggest that miR-24-3p and miR-27a-3p could downregulate the expression of MXI1 gene at post-transcriptional level.
To validate whether MXI1 is directly targeted by miR-24-3p and miR-27a-3p, we mutated the predicted target sites in MXI1 3' UTR of the two miRNAs. The predicted results showed that there was one putative target site for miR-24-3p at MXI1 3' UTR (870-892) and two putative target sites for miR-27a-3p: one was on MXI1 3' UTR (238-258), the other was on MXI1 3' UTR (2352-2374). According to the prediction results, we divided MIX1 3' UTR into two fragments MXI1 3' UTR1 and MXI1 3' UTR2 containing these predicted sites (Fig. 5A), then we cloned these wild-type 3' UTR fragments 
A

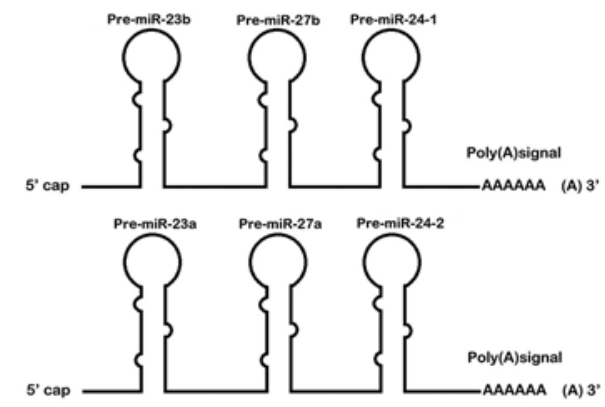

B

\begin{tabular}{|c|c|c|c|c|}
\hline & TargetScan & PicTar & miRanda & EIMMo \\
\hline hsa-miR-24-3p & Yes & Yes & Yes & Yes \\
\hline hsa-miR-24-1-5p & - & - & - & - \\
\hline hsa-miR-24-2-5p & - & - & - & - \\
\hline hsa-miR-27a-3p & Yes & - & Yes & - \\
\hline hsa-miR-27a-5p & - & - & - & - \\
\hline hsa-miR-27b-3p & Yes & - & Yes & - \\
\hline hsa-miR-27b-5p & - & - & - & - \\
\hline
\end{tabular}

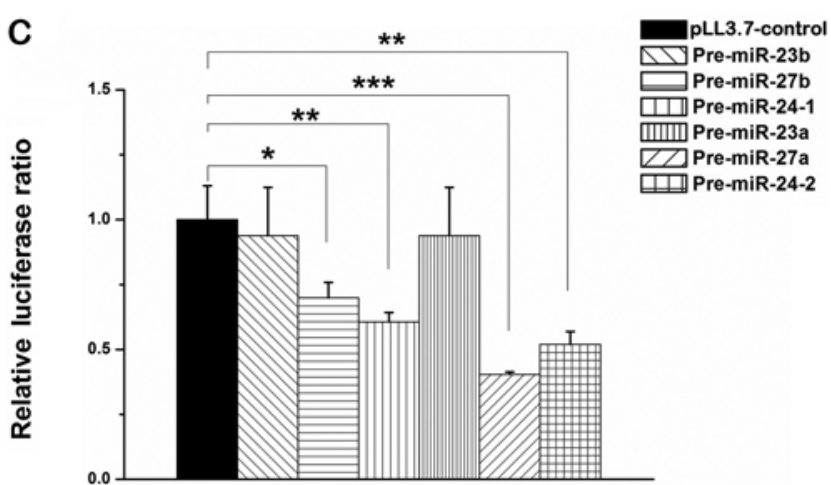

MXI1-3'UTR full

Figure 3. miRNAs from two clusters cooperatively target MXI1 3' UTR. (A) Schematic view of two miRNA clusters miR-23a miR-27a miR-24-2 and miR23b miR-27b miR-24-1. (B) MXI1 3' UTR target prediction performed by prediction software. (C) miRNA expression vectors (pre-miR-24-1, pre-miR-24-2, pre-miR-27a, pre-miR-27b, pre-miR-23a and pre-miR-23b) or empty vector were co-transfected with MXI1 3' UTR in 293T cells, luciferase activity was measured after $24 \mathrm{~h}$. Values represent the means \pm SD from three independent experiments. Significant differences from the control value are indicated by ${ }^{*} \mathrm{p}<0.05,{ }^{* *} \mathrm{p}<0.01$ and $^{* * *} \mathrm{p}<0.001$.

A

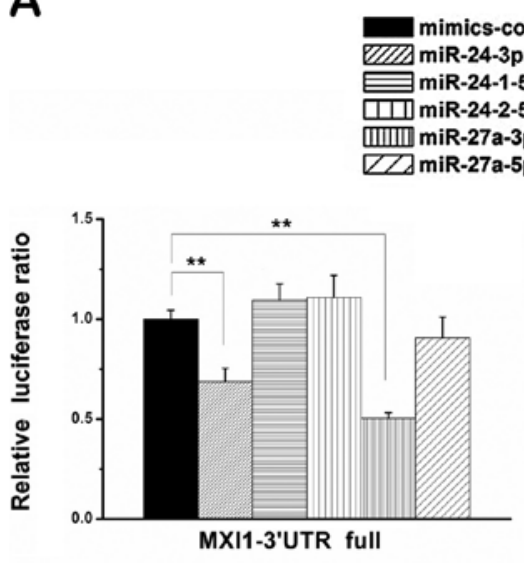

C

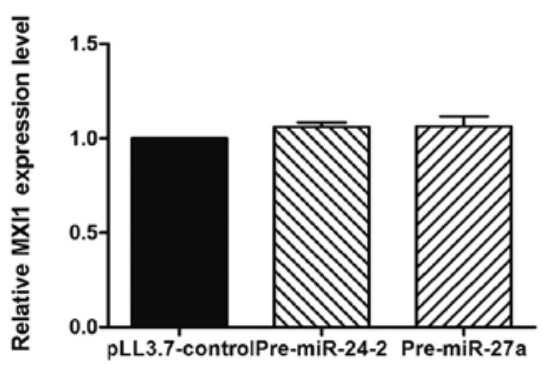

B

$$
\text { pLL3.7- Pre- Pre- }
$$$$
\text { control miR-24-2 miR-27a }
$$

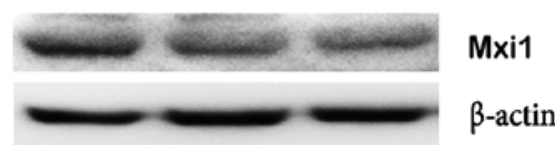

D

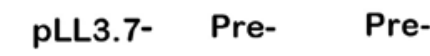

control miR-24-2 miR-27a

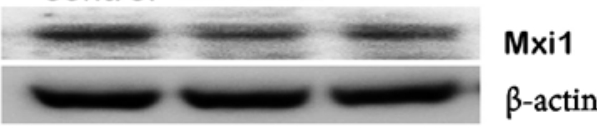

E

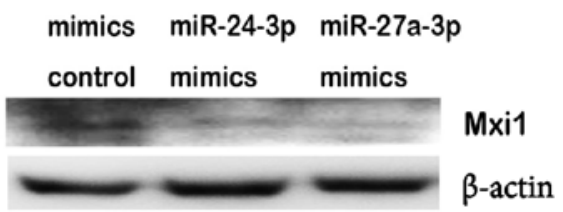

Figure 4. miR-24-3p and miR-27a-3p interacting with MXI1 3' UTR were further confirmed. (A) miRNA (miR-24-3p, miR-24-1-3p, miR-24-2-5p miR-27a-3p or miR-27a-5p) mimics or negative control (NC) was co-transfected with MXI1 3' UTR full in HEK293T cells, luciferase activity was measured $24 \mathrm{~h}$ after transfection. Each data point was measured in triplicate. Values represent the means \pm SD. Significant differences from the control value are indicated by ${ }^{* *} \mathrm{p}<0.01$. (B) Western blot analysis of Mxil protein levels after introduction of miRNA expression vectors (pre-miR-24-2 and pre-miR-27a) in 293T cells. $\beta$-actin served as the internal control. (C) Relative expression level of MXI1 in 293T cells measured by qRT-PCR. (D) Western blot analysis of Mxil protein levels after introduction of miRNA expression vectors (pre-miR-24-3p and pre-miR-27a-3p) in U87 cells. $\beta$-actin served as the internal control. (E) Western blot analysis of Mxil protein levels after introduction of miR-24-3p and miR-27a-3p mimics in U87 cells. $\beta$-actin served as the internal control. 


\section{A}

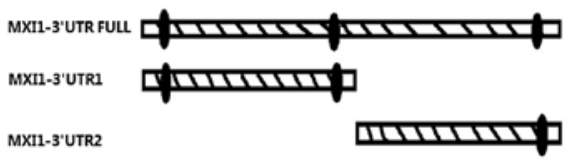

miR-27a-3P miR-24a-3p miR-27a-3P
C

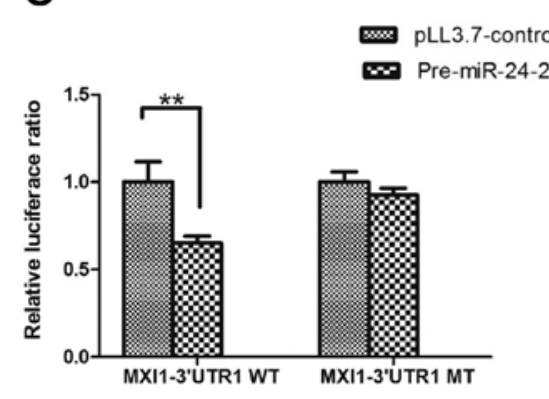

E

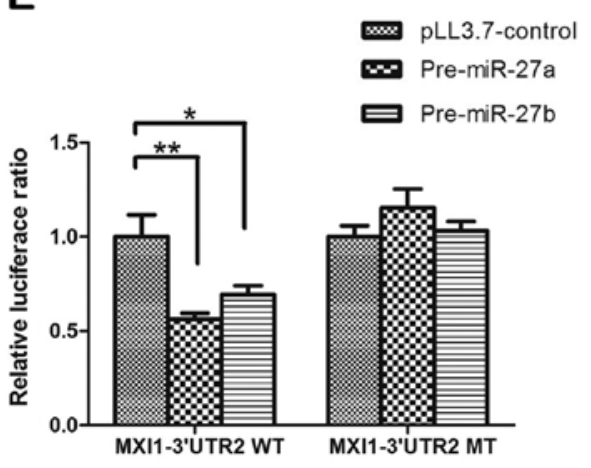

B

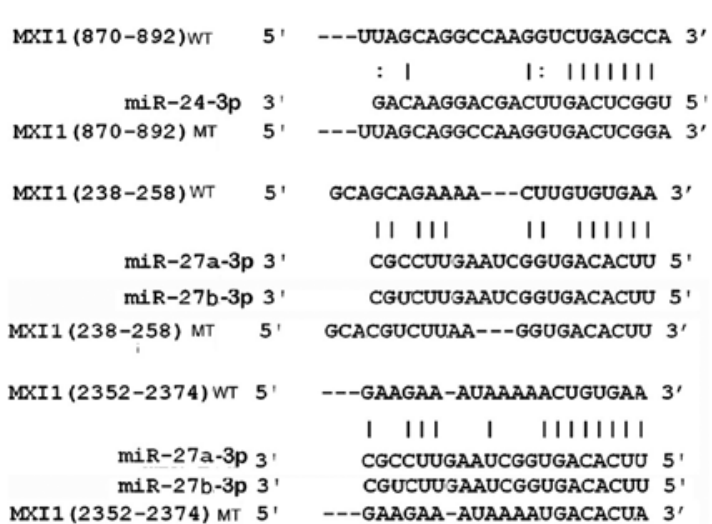

D

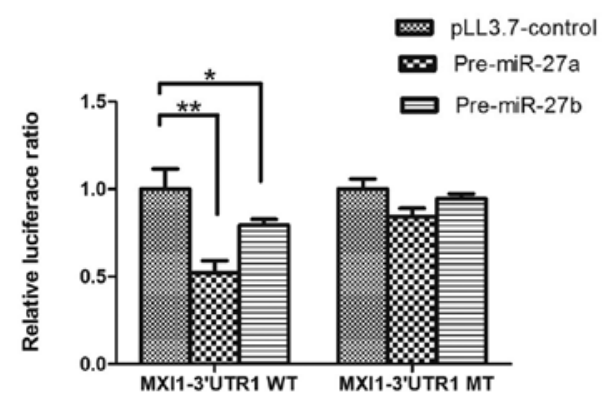

$\mathbf{F}$

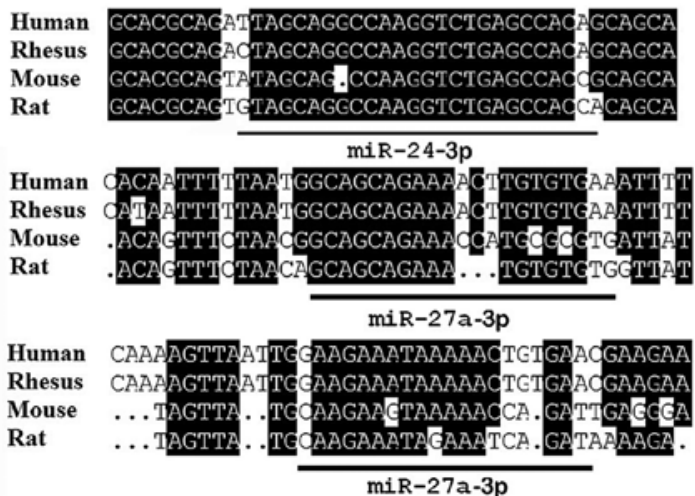

Figure 5. MXI1 is the direct downstream target of miR-24-3p, miR-27a-3p and miR-27b-3p. (A) Schematic diagram of the binding sites of miR-24-3p, miR-27a-3p and miR-27b-3p with wild-type MXI1 3' UTR. MXI1 3' UTR full length was divided into two parts, UTR1 and UTR2. (B) Three predicted target sequences of miR-24-3p, miR-27a-3p and miR-27b-3p within the $3^{\prime}$ UTR of MXI1. Several nucleotides within the seed region were mutated in the 3' UTR of MXI1. (C, D and E) Empty vector or miRNA expression vector (pre-miR-24-2, pre-miR-27a or pre-miR-27b) was co-transfected with wild type UTR1, UTR2 or mutant UTR1 and UTR2. Luciferase acivities were measured at $48 \mathrm{~h}$ post-transfection. Each data point was measured in triplicate. Values represent the means \pm SD. Significant differences from the control value are indicated by ${ }^{*} \mathrm{p}<0.05$ and ${ }^{* *} \mathrm{p}<0.01$. (F) Alignment of human, rhesus, mouse and rat MXI1 3' UTR binding sites of miR-24-3p and miR-27a-3p.

into the psiCHECK-2 luciferase reporter vectors separately. The mutants within binding regions were also cloned as shown in Fig. 5B. The results of luciferase ratios indicated that pre-miR-24-2 could downregulate MXI1 3' UTR1, pre-miR-27a could downregulated both MXI1 3' UTR1 and MXI1 3' UTR2 (Fig. 5C-E). Also, pre-miR-27b has similar effect to the pre-miR-27a (Fig. 5D and E). By contrast, the repressive effect of these two miRNAs on luciferase activity was abrogated by mutations in the MXI1 3' UTR fragments (Fig. 5C-E). In addition, we found that the target sites are quite conserved when aligning the sequences from human, rhesus, rat and mouse (Fig. 5F). Taken together, all these results suggest that MXI1 is a direct downstream target of miR-24-3p, miR-27a-3p and miR-27b-3p.

MXI1 rescues the effects of $m i R-24-3 p$ and $m i R-27 a-3 p$ in cell proliferation. MXI1 is a tumor suppressor gene involved in regulation of cell proliferation (34). Especially, overexpression of MXI1 in glioma cells is capable of inhibiting cell proliferation (Fig. 6A). To better understand the role of miR-24-3p and miR-27a-3p with MXI1 in regulating cell proliferation, we constructed the MXI1 expression vector by inserting 

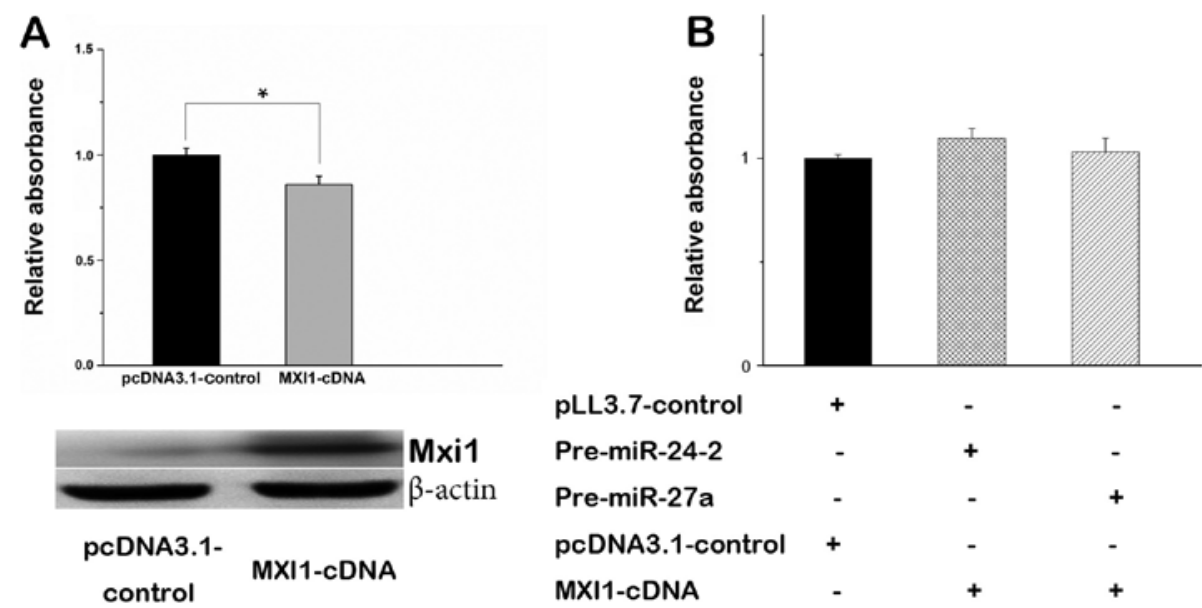

Figure 6. MXI1-cDNA could rescue the effect of miR-24-3p or miR-27a-3p. (A) U87 cells were transfected with empty vector or with MXI1-cDNA and the cell growth was investigated by MTT assay after $72 \mathrm{~h}$. Western blot analysis of Mxil protein levels after introduction of MXI1-cDNA (pre-miR-24-2 and pre-miR-27a) in U87 cells. $\beta$-actin served as the internal control. Significant differences from the control value are indicated by "p<0.05. (B) Co-transfection of pre-miR-24-2 or pre-miR-27a expression vector with MXI1-cDNA into U87 cells, the cell growth was investigated by MTT assay after $72 \mathrm{~h}$. Absorbance at $570 \mathrm{~nm}$ was normalized to the control to determine cell viability. Values represent the means $\pm \mathrm{SD}$.

A

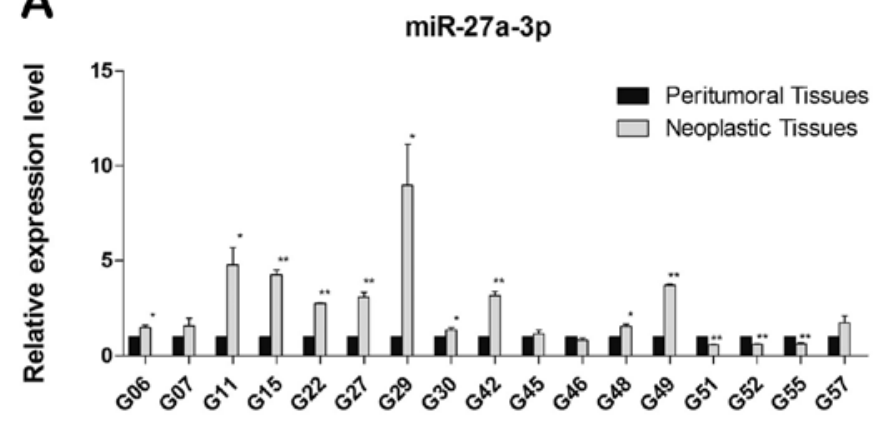

C

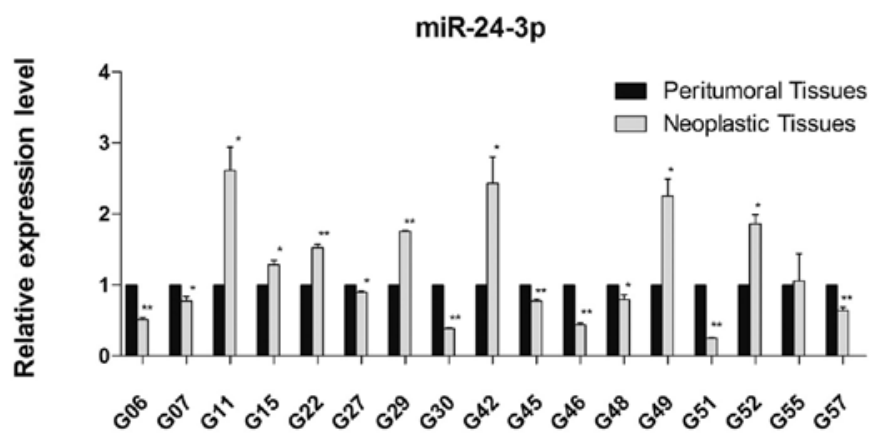

B

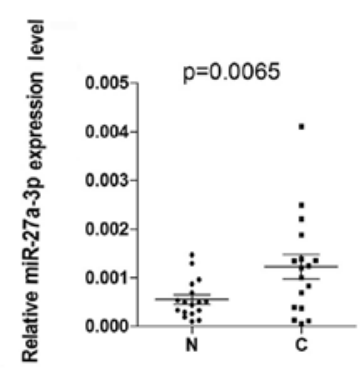

Figure 7. Expression of miR-24-3p or miR-27a-3p in tumor or peritumoral brain tissues. (A) Expression of miRNA-27a-3p in 17 pairs of tumor samples (gray bars) measured relative to peritumoral tissues (black bars). (B) Densitometry of pooled samples of miR-27a-3p was measured in neoplastic tissues (C) compared with peritumoral tissues $(\mathrm{N})$. (C) Expression of miRNA-24 -3p in 17 pairs of tumor samples (gray bars) was measured relative to peritumoral tissues (black bars). (D) Densitometry of pooled samples of miR-24a-3p was measured in neoplastic tissues compared with peritumoral tissues.

MXI1-cDNA without 3' UTR into the pcDNA3.1 vector. The MTT assays showed that transient transfection of MXI1 expression vector in U87 cells significantly decreased cell proliferation (Fig. 6A). When MXI1 gene without 3' UTR was co-transfected with pre-miR-24-2 or pre-miR-27a, the effect of miR-24-3p or miR-27a-3p in cell proliferation was rescued (Fig. 6B), suggesting that MXI1 is an antagonist of miR-24-3p or miR-27a-3p. 
miR-27a-3p is upregulated in human neoplastic brain tissues. We experimentally tested miR-24-3p and miR-27a-3p involved in neoplastic brain tissues and peritumoral brain tissues from GBM patient samples, in which qRT-PCR were used to analyze the expression of miR-27a-3p and miR-24-3p, and expression levels were normalized to those of U6 RNA used as the internal control. Compared with neoplastic brain tissues and peritumoral brain tissues, we observed that expression of miR-27a-3p in neoplastic brain significantly increased in 10 of 17 pairs of samples, the expression of miR-27a-3p decreased in 3 pairs of samples and 4 pairs have no significant difference $\left(\mathrm{p}<0.05 ;{ }^{* *} \mathrm{p}<0.01\right)$ (Fig. 7A). Densitometry of tissue samples revealed that miR-27a-3p was significantly induced about 3 -fold $(p=0.0065)$ in neoplastic tissues compared with peritumoral tissues (Fig. 7B). However, while testing miR-24-3p, the results did not show significant difference between the two tissues (Fig. 7C), with the densitometry of neoplastic and peritumoral brain tissue samples equal to.1.3-fold ( $\mathrm{p}=0.3103$ ) (Fig. 7D).

\section{Discussion}

Oligonucleotides have potential to be widely used in diagnostics and therapeutics. They have many advantages such as easy production, high target selectivity and stability (37). Oligonucleotide-based approaches, including miRNA and siRNA, may provide a strategy for cancer therapy. miRNAs play an important role in cell cycle control, differentiation, proliferation and apoptosis (38), and deregulation of miRNA expression has been observed in glioma tumors compared with normal tissues, to find the miRNAs involved in tumorgenesis will be an important issue in identification of the therapeutical targets. miR-24-3p and miR-27a-3p were upregulated in glioma tissues or cell lines $(15,39)$ and several studies have reported that miR-24-3p might regulate cell proliferation in different types of cells $(11,16,17)$, it is important to study the function of these miRNAs in glioma cells. In this study, we demonstrated that both miR-24-3p and miR-27a-3p promoted cell proliferation in glioma cells.

miR-24-3p is a master regulator in gene regulation (11) and plays a critical role in diverse physiological process. miR-27a-3p has also been reported to regulate several genes including FOXO-1 in breast cancer cells (40), MDR1/Pglycoprotein in multidrug resistant (MDR) cancer cells lines (41) and RXR $\alpha$ in adipogenesis (22). However, the synergetic regulation by the two miRNAs from this cluster is poorly characterized. In the present study, we report for the first time that miR-24-3p and miR-27a-3p, as well as miR$27 b-3 p$ have been found to regulate the same gene MXI1 in 293T and glioma cells. Several lines of evidence support a direct interaction between the three miRNAs and the MXI1 3' UTRs. Firstly, the human MXI1 3' UTR contains one putative miR-24-3p binding site and two putative miR-27a/b-3p binding sites with prominent seed matches (Fig. 5A and B). Secondly, miR-24-3p, miR-27a-3p and miR-27b-3p can suppress the activity of luciferase reporter genes fused to the 3' UTR of MXI1 mRNA. While the target sites were mutated, no significant changes have been shown in luciferase activities. Thirdly, the miRNA precursors pre-miR-24-2 and pre-miR$27 a$ or synthetic mimics of miR-24-3p and miR-27a-3p are able to repress endogenous expression of human MXI1 in 293T cells and U87 glioma cells at the protein level. Previous studies reported that overexpression of MXI1 in glioma cells suppressed cell proliferation and promote cell differentiation (42). We observed that cell proliferation increased when miR-24-3p or miR-27a-3p was overexpressed in U87 and U251 glioma cells, which suggests that miR-24-3p or miR-27a-3p affects cell proliferation in glioma cells via downregulation of MXI1. This hypothesis was confirmed by the rescue experiments. Overexpression of MXI1 without the 3' UTR, which the miR-24-3p or miR-27a-3p has no effect on, inhibited the ability of miR-24-3p or miR-27a-3p to promote cell proliferation in U87 glioma cells. This study provides the first identification and demonstration of three miRNAs from the two miRNA clusters miR-23a miR-27a miR-24-2 and miR-23b miR-27b miR-24-1 which directly regulate MXI1 and this in turn enhances our understanding of the function and regulation of MXI1 in glioma cells.

MXI1 plays an important role in oncogenesis (43). MXI1 gene alterations could be involved in the development and/ or the progression of prostate cancer (44). Loss of MXI1 function contributes to renal cystic disease (43). Abnormal cell proliferation and tumorigenesis have been observed in organs of MXI1-/- mice (34). MXI1 also plays an important role in hepatocyte proliferation (45). MXI1 is a member of the Mad family, the interaction between MXI1 and Max could antagonize Myc oncoproteins and thereby suppress glioma progression (46). For cancer therapies, Myc proteins are validated targets (47). However, because of the difficulty to target the transcription factors that lack clear binding domains, targeting Myc directly is not yet a treatment option (46). In contrast, small molecular drugs that inhibit Myc-Max interactions are effective (46). For this reason, we provide a clue that inhibition of miR-24-3p and miR-27a-3p upregulated MXI1, which then inhibited Myc protein level and finally inhibited the glioma cell proliferation (12).

It is documented that all three miRNAs of this cluster are derived from a single primary transcript (25), however, different biological conditions makes the expression pattern varied (11). Sometimes all three miRNAs have similar expression patterns, but in some cases one or two of the three miRNAs are expressed and the third is not (11). This might be the reason that miR-27a-3p is upregulated in glioma tissues, however, there was no significant difference for miR-24-3p between neoplastic brain tissues and peritumoral brain tissues.

This study demonstrates the role of miR-24-3p and miR-27a-3p in promoting cell proliferation of glioma cells. Their function was realized partially via its downstream target MXI1. It is likely that inhibition of miR-24-3p and miR-27a-3p could serve in prevention of gliomas. Our studies shed light on developing therapeutic strategies against gliomas by abrogating the level of miR-24-3p and miR-27a-3p and increasing MXI1.

\section{Acknowledgements}

This study was supported by grants from the National Natural Science Foundation of China (grant no. 81272773 and 81101960); the Scientific and Technological Planning of Guangzhou (grant no. 2012J4100082) and the Fundamental 
Research Funds for the Central Universities (grant no. 101gpy23).

\section{References}

1. Surawicz TS, Davis F, Freels S, Laws ER Jr and Menck HR: Brain tumor survival: results from the National Cancer Data Base. J Neurooncol 40: 151-160, 1998.

2. Furnari FB, Fenton T, Bachoo RM, et al: Malignant astrocytic glioma: genetics, biology, and paths to treatment. Genes Dev 21: 2683-2710, 2007.

3. Xu X, Xi L, Zeng J and Yao Q: A functional $+61 \mathrm{G} / \mathrm{A}$ polymorphism in epidermal growth factor is associated with glioma risk among Asians. PLoS One 7: e41470, 2012.

4. Lu J, Getz G, Miska EA, et al: MicroRNA expression profiles classify human cancers. Nature 435: 834-838, 2005.

5. Dong H, Siu H, Luo L, Fang X, Jin L and Xiong M: Investigation gene and microRNA expression in glioblastoma. BMC Genomics 11 (Suppl 3): S16, 2010.

6. Chen CZ: MicroRNAs as oncogenes and tumor suppressors. N Engl J Med 353: 1768-1771, 2005

7. Calin GA and Croce CM: MicroRNA signatures in human cancers. Nat Rev Cancer 6: 857-866, 2006.

8. Bushati N and Cohen SM: microRNA functions. Annu Rev Cell Dev Biol 23: 175-205, 2007.

9. Bartel DP: MicroRNAs: target recognition and regulatory functions. Cell 136: 215-233, 2009.

10. Wang D, Qiu C, Zhang H, Wang J, Cui Q and Yin Y: Human microRNA oncogenes and tumor suppressors show significantly different biological patterns: from functions to targets. PLoS One 5: e13067, 2010.

11. Chhabra R, Dubey R and Saini N: Cooperative and individualistic functions of the microRNAs in the miR-23a 27a 24-2 cluster and its implication in human diseases. Mol Cancer 9: 232, 2010.

12. Lal A, Navarro F, Maher CA, et al: miR-24 inhibits cell proliferation by targeting E2F2, MYC, and other cell-cycle genes via binding to 'seedless' 3'UTR microRNA recognition elements. Mol Cell 35: 610-625, 2009.

13. Lal LS, Miller LA, Arbuckle R, et al: Disparities in outpatient antidepressant prescribing patterns and determinants of resource utilization at a tertiary care cancer center. J Support Oncol 7: 237-244, 2009.

14. Jones KJ, Skinner A, Xu L, Sun J and Mueller K: The AHRQ Hospital Survey on Patient Safety Culture: A Tool to Plan and Evaluate Patient Safety Programs. In: Advances in Patient Safety: New Directions and Alternative Approaches Henriksen K, Battles JB, Keyes MA, Grady ML (eds). Vol 2. Culture and Redesign. Agency for Healthcare Research and Quality, Rockville, MD, 2008.

15. Fukuda Y, Kawasaki $\mathrm{H}$ and Taira K: Exploration of human miRNA target genes in neuronal differentiation. Nucleic Acids Symp Ser (Oxf): 341-342, 2005.

16. Qin W, Shi Y, Zhao B, et al: miR-24 regulates apoptosis by targeting the open reading frame (ORF) region of FAF1 in cancer cells. PLoS One 5: e9429, 2010.

17. Cheng AM, Byrom MW, Shelton J and Ford LP: Antisense inhibition of human miRNAs and indications for an involvement of miRNA in cell growth and apoptosis. Nucleic Acids Res 33 1290-1297, 2005.

18. Feng J, Iwama A, Satake M and Kohu K: MicroRNA-27 enhances differentiation of myeloblasts into granulocytes by post-transcriptionally downregulating Runx1. Br J Haematol 145: 412-423, 2009.

19. McDaneld TG, Smith TP, Doumit ME, et al: MicroRNA transcriptome profiles during swine skeletal muscle development. BMC Genomics 10: 77, 2009.

20. Wang $\mathrm{T}$ and $\mathrm{Xu} \mathrm{Z}$ : miR-27 promotes osteoblast differentiation by modulating Wnt signaling. Biochem Biophys Res Commun 402: $186-189,2010$.

21. Mertens-Talcott SU, Chintharlapalli S, Li X and Safe S: The oncogenic microRNA-27a targets genes that regulate specificity protein transcription factors and the G2-M checkpoint in MDA-MB-231 breast cancer cells. Cancer Res 67: 11001-11011, 2007.

22. Ji J, Zhang J, Huang G, Qian J, Wang X and Mei S: Overexpressed microRNA-27a and $27 \mathrm{~b}$ influence fat accumulation and cell proliferation during rat hepatic stellate cell activation. FEBS Lett 583: 759-766, 2009.
23. Lee Y, Kim M, Han J, et al: MicroRNA genes are transcribed by RNA polymerase II. EMBO J 23: 4051-4060, 2004.

24. Sun F, Wang J, Pan Q, et al: Characterization of function and regulation of miR-24-1 and miR-31. Biochem Biophys Res Commun 380: 660-665, 2009.

25. Ciafrè SA, Galardi S, Mangiola A, et al: Extensive modulation of a set of microRNAs in primary glioblastoma. Biochem Biophys Res Commun 334: 1351-1358, 2005.

26. Landgraf $\mathrm{P}$, Rusu M, Sheridan R, et al: A mammalian microRNA expression atlas based on small RNA library sequencing. Cell 129: 1401-1414, 2007.

27. Henriksson M and Lüscher B: Proteins of the Myc network: essential regulators of cell growth and differentiation. Adv Cancer Res 68: 109-182, 1996.

28. Schreiber-Agus N and DePinho RA: Repression by the Mad(Mxi1)-Sin3 complex. Bioessays 20: 808-818, 1998.

29. Fujimoto M, Fults DW, Thomas GA, et al: Loss of heterozygosity on chromosome 10 in human glioblastoma multiforme. Genomics 4: 210-214, 1989.

30. Rasheed BK, Fuller GN, Friedman AH, Bigner DD and Bigner SH: Loss of heterozygosity for $10 \mathrm{q}$ loci in human gliomas. Genes Chromosomes Cancer 5: 75-82, 1992.

31. Ransom DT, Ritland SR, Moertel CA, et al: Correlation of cytogenetic analysis and loss of heterozygosity studies in human diffuse astrocytomas and mixed oligo-astrocytomas. Genes Chromosomes Cancer 5: 357-374, 1992.

32. Carter BS, Ewing CM, Ward WS, et al: Allelic loss of chromosomes 16q and 10q in human prostate cancer. Proc Natl Acad Sci USA 87: 8751-8755, 1990

33. Lundgren R, Mandahl N, Heim S, Limon J, Henrikson H and Mitelman F: Cytogenetic analysis of 57 primary prostatic adenocarcinomas. Genes Chromosomes Cancer 4: 16-24, 1992.

34. Ko JY, Yoo KH, Lee HW and Park JH: Mxi1 regulates cell proliferation through insulin-like growth factor binding protein-3. Biochem Biophys Res Commun 415: 36-41, 2011.

35. Tsao CC, Teh BT, Jonasch E, et al: Inhibition of Mxi1 suppresses HIF-2alpha-dependent renal cancer tumorigenesis. Cancer Biol Ther 7: 1619-1627, 2008.

36. Ebert MS, Neilson JR and Sharp PA: MicroRNA sponges: competitive inhibitors of small RNAs in mammalian cells. Nat Methods 4: 721-726, 2007.

37. Catuogno S, Esposito CL, Quintavalle C, Condorelli G, de Franciscis V and Cerchia L: Nucleic acids in human glioma treatment: innovative approaches and recent results. J Signal Transduct 2012: 735135, 2012.

38. Esquela-Kerscher A and Slack FJ: Oncomirs - microRNAs with a role in cancer. Nat Rev Cancer 6: 259-269, 2006.

39. Feng SY, Dong CG, Wu WK, Wang XJ, Qiao J and Shao JF: Lentiviral expression of anti-microRNAs targeting miR-27a inhibits proliferation and invasiveness of U87 glioma cells. Mol Med Rep 6: 275-281, 2012.

40. Guttilla IK and White BA: Coordinate regulation of FOXO1 by miR-27a, miR-96, and miR-182 in breast cancer cells. J Biol Chem 284: 23204-23216, 2009.

41. Zhu H, Wu H, Liu X, et al: Role of MicroRNA miR-27a and miR-451 in the regulation of MDR1/P-glycoprotein expression in human cancer cells. Biochem Pharmacol 76: 582-588, 2008.

42. Wechsler DS, Shelly CA, Petroff CA and Dang CV: MXI1, a putative tumor suppressor gene, suppresses growth of human glioblastoma cells. Cancer Res 57: 4905-4912, 1997.

43. Schreiber-Agus N, Meng Y, Hoang T, et al: Role of Mxi1 in ageing organ systems and the regulation of normal and neoplastic growth. Nature 393: 483-487, 1998.

44. Kuczyk MA, Serth J, Bokemeyer C, et al: The MXI1 tumor suppressor gene is not mutated in primary prostate cancer. Oncol Rep 5: 213-216, 1998.

45. Mauleon I, Lombard MN, Muñoz-Alonso MJ, Cañelles M and Leon J: Kinetics of myc-max-mad gene expression during hepatocyte proliferation in vivo: Differential regulation of mad family and stress-mediated induction of c-myc. Mol Carcinog 39: 85-90, 2004.

46. Swartling FJ: Myc proteins in brain tumor development and maintenance. Ups J Med Sci 117: 122-131, 2012.

47. Gustafson WC and Weiss WA: Myc proteins as therapeutic targets. Oncogene 29: 1249-1259, 2010. 